\title{
ARTIGOS
}

\section{DOUTE METHODIQUE ET MORALE CARTESIENNE}

Jean-Robert Armogathe

RESUMO $O$ artigo examina o estatuto da moral ao longo da obra de Descartes, da «moral por provisão» do Discurso à «moral como sabedoria» da carta-prefácio à edição francesa dos Princípios. A constituição da metafísica neste interim, com a separação entre a verdade e o bem, permite a Descartes tomar a «moral como sabedoria» como perfeita e definitiva não obstante a dúvida epistemológica que esta moral traz em seu âmago.

ABSTRACT The article examines the place and nature of morals in Descartes's works, from the "provisional morals » of the Discourse to the conception of morals as wisdom in the letter-preface to the French edition of the Principles. Descartes's elaboration of his metaphysics, with the separation of the truth from the good, allows him to assume his later view of morals as wisdom as definitive and perfect despite the epistemic doubt intrinsic to this morals.

Palavras-chave: Descartes, moral, dúvida, modernidade

“Je puis dire, avec vérité, que la principale règle que j'ai toujours observée en mes études, et celle que je crois m'avoir le plus servi pour acquérir quelque connaissance, été que je n'ai jamais employé que fort peu d'heures, par jour, aux pensées qui occupent l'imagination, et fort peu d'heures, par an, à celles qui occupent l'entendement seul, et que j'ai donné tout le reste de

* E.P.H.E. (Sciences Religieuses), França.

KRITERION, Belo Horizonte, n 106, Dez/2002, p.9-19 
mon temps au relâche des sens et au repos de l'esprit; même je compte, entre les exercices de l'imagination, toutes les conversations sérieuses, et tout ce à quoi il faut avoir de l'attention" : en ces termes, Descartes expose en 1643 à la princesse Elisabeth le programme de vie qui fut le sien. Loin d'être envahi par la recherche des sciences, qui occupe l'imagination, et la réflexion métaphysique, qui occupe l'entendement seul, Descartes a placé au cœur de son expérience le souci de la morale, la recherche de la sagesse. L'entreprise métaphysique ne peut pas être prise à la légère, et la publication, en 1637, du Discours de la méthode suivi des trois Essais, est une démarche profondément volontaire de la part de cet homme de quarante et un ans. Or dans l'exposition de l'effort initial, méthodique, destiné á écarter tout préjugé, dans l'institution de ce doute hyperbolique, opération mentale qui lui semble indispensable pour progresser dans la recherche de la vérité, pour voir clair dans ses actions et marcher avec assurance en cette vie (AT 6, 10), ${ }^{2}$ Descartes entend expressément préserver un espace d'action, et par conséquent une morale de l'agir.

C'est un lieu commun de rappeler comment Descartes aménage cet espace immune du doute méthodique, qu'il nomme morale par provision, dont il met à part, avec les vérités de foi, trois ou quatre maximes:

afin que je ne demeurasse point irrésolu en mes actions pendant que la raison m'obligerait de l'être en mes jugements, ${ }^{3}$ et que je ne laissasse pas de vivre dès lors le plus heureusement que je pourrais, je me formai une morale par provision, qui ne consistait qu'en trois ou quatre maximes, dont je veux bien vous faire part (AT 6, 22).

«Dont je veux bien vous faire part » (ou, dans la traduction latine du Discours : «non pigebit adscribere »): une nette réserve est ainsi marquée par ce caractère confidentiel, sorte d'aveu d'une morale possible. En 16471648, c'est-à-dire dans le contexte des attaques dont il est objet de la part des Jésuites en France et des ministres réformés en Hollande, Descartes explique à Chanut ${ }^{4}$ et à Burman ${ }^{5}$, les raisons de sa prudence et de son aveu :

1 Nous désignons l'édition des Oeuvres de Descartes par Charles Adam et Paul Tannery, révisée et complétée par J. Beaude, P. Costabel et R. Rochot par AT suivi du tome et de la page. Ici lettre du 26 juin 1643, AT 3, 693.

2 «ut rectum iter vitae clarius viderem, et majori cum securitate persequerer» (De methodo, AT 6, 545).

3 Dans son commentaire, Ferdinand Alquié donne le sens exact de la distinction proposée entre " mes actions » et «mes jugements »: «être irrésolu dans ses jugements, ce n'est pas s'abstenir de juger » (Oeuvres philosophiques, t. 1, p. 594).

4 Lettre du 20 novembre 1647, AT 5, 86.

5 Entretien AT 5, 178 ; Ed. Beyssade, Paris, PUF, 1981, p. 145. 
auctor non libenter scribit ethica, sed propter paedagogos et similes coactus est has regulas adscribere, quia alias dicerent illum esse sine religione, fide, et per suam methodum haec evertere velle.

Etienne Gilson a admirablement commenté ces trois règles, et les approchements avec Charron et Montaigne sont éclairants, nous y reviendrons. Il demeure que les maximes que Descartes énonce dans cette troisième partie du Discours de la méthode semblent fort banales: obéissance à la tradition, en religion comme en politique, fidélité aux résolutions prises et restriction de nos désirs à ce qui nous est accessible. Elles constituent un ensemble cohérent pour l'action, mais qui n'appelle ni à l'héroïsme ni à la sainteté. Il s'agit bien d'une morale par provision, à condition de bien entendre ce que signifie l'expression.

Geneviève Rodis-Lewis a rapproché du syntagme «par provision » une phrase du Père Richeome (d'un ouvrage, Le pèlerin de Lorette, paru en 1603, Oeuvres t. 2, p. 200a, que Descartes a bien pu lire) :

«en toute action projetée avec prudence, il est requis de (...) faire provision de moyens propres pour y parvenir» ${ }^{6}$.

A cette référence, je pense qu'il faut joindre le sens juridique de l'expression et, surtout, prendre en considération le syntagme par provision: l'Inventaire de Philibert Monet (Lyon, 1635) donne comme synonymes de «par provision », «pour commencer, en attendant $»^{7}$. Descartes écrit ailleurs, dans le même sens:

une Morale imparfaite, qu'on peut suivre par provision pendant qu'on n'en sait point encore de meilleure ${ }^{8}$.

Descartes propose à Huygens, en 1637, «un Abrégé de médecine, que je tire en partie des livres, et en partie de mes raisonnements, duquel j'espère me pouvoir servir par provision à obtenir quelque délai de la Nature, et ainsi poursuivre mieux ci-après en mon dessein» (4 décembre 1637, AT I, 649). Il écrit à Reneri pour Pollot (1638, AT I, 35) :

je ne me sers [d'une maxime] que par provision, avec dessein de changer mes opinions, sitôt que j'en pourrai trouver de meilleures, et de ne perdre aucune occasion d'en chercher.'

6 Cité dans Gen. Rodis-Lewis, L'oeuvre de Descartes, Paris, J. Vrin, 1971, p. 454.

7 Philibert Monet Inventaire des deus langues françoise et latine, assorti des plus utiles curiositez de l'un et de l'autre idiomes, Lyon, 1635, réimpr. de l'Ėd. 1636 chez Slatkine, Genève (1973).

8 Principes de philosophie, lettre-préface, AT 9,15.

9 Voir encore AT 2, 268 («par provision je vous dirai...»); 3, 47 («si vous attendez que je vous dise par provision ma conjecture...»); 3, 128 («et par provision je serai bien aise qu'il soit su de tous ceux auxquels il vous plaira montrer ma réponse». 
Il s'agit bien d'une morale " pour commencer », de ce qui permet tout à la fois d'attendre et de mettre en oeuvre sans attendre: ce double sens souligne toute l'ambiguïté d'une décision qui est à la fois moratoire et initiale.

La séquence historico-génétique des textes permet de relire la constitution de la morale cartésienne dans la complexité de son intrication métaphysique, depuis la «morale par provision » du Discours de la méthode jusqu' aux échanges sur la sagesse des dernières années. La période 1643-1650 est complexe: on peut difficilement dissocier la correspondance avec Elisabeth, Chanut et Christine de la rédaction contemporaine de deux grands traités : celui des Passions, rédigé pendant l'hiver 1645-1646, mais remanié et publié par Descartes en 1649 seulement, celui des Principia/Principes, dont la préface latine à Elisabeth (1644) et la lettre-préface de l'édition française (1646) touchent de près à la morale. Les «trois morales de Descartes » : prudence, sagesse et générosité ${ }^{10}$ ne peuvent donc pas être classées dans une succession chronologique stricte, et les pages des Passions sur la générosité sont antérieures à la lettre-préface sur la sagesse ${ }^{11}$.

En 1647, en effet, Descartes définit la sagesse par la seule connaissance:

par la sagesse, on n'entend pas seulement la prudence dans les affaires, mais une parfaite connaissance de toutes les choses que l'homme peut savoir, tant pour la conduite de sa vie que pour la conservation de sa santé et l'invention de tous les arts.

La suite du texte distingue encore une sagesse incertaine (celle des opinions, celle des lectures) et une sagesse véritable, liée à la connaissance vraie et certaine. Cette insistance sur le lien entre sagesse et connaissance parfaite n'est pourtant, dans le texte latin (lettre-préface à Elisabeth de 1644), qu'une seule des deux voies:

Et quamvis sufficere debeat iis qui sunt natura tardiusculi, quod, etsi multa ignorent, modo tamen firmam et constantem retineant voluntatem nihil omittendi, quo ad recti cognitionem perveniant, atque id omne quod rectum judicabunt exsequendi, pro modulo suo sapientes et hoc nomine Deo gratissimi esse possint: multo tamen praestantiores illi sunt, in quibus, cum firmissima recte agendi voluntate, perspicacissimum ingenium et summa veritatis cognoscendae cura reperitur. ${ }^{12}$

10 Sous-titre et titre de la communication de T. Ruyssen au Congrès de Bordeaux Les sciences et la sagesse, 1950, p. 235-238 (référence donnée par Geneviève Rodis-Lewis, La morale de Descartes p. 5).

11 Lettre d'Elisabeth 25 avril 1646 (AT 4, 404), où elle se dit fort satisfaite de «toute la partie morale » du premier manuscrit du traité (des Passions) que Descartes lui avait envoyé.

12 AT 8-1, 3. Traduction française de l'abbé Picot : «mais, encore que ceux qui n'ont pas le plus d'esprit puissent être aussi parfaitement sages que leur nature le permet, et se rendre très agréables à Dieu par leur vertu, si seulement ils ont toujours une ferme résolution de faire tout le bien qu'ils sauront, et de n'omettre rien pour apprendre celui qu'ils ignorent; toutefois ceux qui, avec une constante volonté de bien faire et un soin très particulier de s'instruire, ont aussi un très excellent esprit, arrivent sans doute à un plus haut degré de sagesse que les autres» (AT 9-2, 23). 
Le doute méthodique épargne les maximes morales que Descartes établit. Mais le doute reste-t-il étranger au projet d'une morale parfaite ? Comment peut-elle se constituer, au long des années ? Et quel statut lui accorder dans le projet cartésien d'un monde nouveau? L'examen des passions permet d'approcher la conversion de la morale à la sagesse. Loin d'être invalidée par le scepticisme philosophique, la morale cartésienne se trouve être à la fois, dans ses états successifs, héritière de la réflexion des Anciens et porteuse des exigences métaphysiques de l'age moderne. Sous le regard de la raison, elle confère à la quête de la sagesse le statut souverain de la conscience.

Descartes commence son enseignement moral par correspondance en commentant le De uita beata de Sénèque (21 juillet 1645$)$, mais très vite il s'irrite du peu d'exactitude que suit l'auteur latin dans sa méthode (AT 3, 264) et il lui oppose «(son) opinion »: il faut analyser les causes du contentement. Il faut chercher « toutes les principales vérités dont la connaissance est requise pour faciliter l'usage de la vertu et régler nos désirs et nos passions, et ainsi jouir de la béatitude naturelle $» .^{13}$

Nous relevons ici encore les deux fonctions que Descartes assigne à la démarche morale: faciliter l'usage de la vertu (et nous sommes encore ici dans un domaine "par provision»), mais aussi (et nous entrons dans le domaine du définitif) régler nos désirs et nos passions. C'est l'union de la vertu pratiquée, de la vertu sans peine, de la vertu sans larmes, et de ses désirs, et de ses passions réglées et contenues. Cette union permet la jouissance de la béatitude naturelle : il s'agit d'un texte français, mais le latin est tout proche, et il faut rappeler que deux mots latins coïncident au $17^{\text {Ëme }}$ siècle, beatitudo et beatitas, que l'on trouve chez Cicéron et chez saint Augustin. Ce couple permet de jouer entre deux types de béatitude (on trouve e scripturis beatitas chez un auteur calviniste de la fin du $16^{\text {ème }}$ siècle).$^{14}$

La morale peut être affectée par le doute de deux manières : d'abord, on peut y étudier l'extension du doute méthodique et en repérer, dans ces conditions, le développement. Mais aussi on peut envisager que le doute appartienne au contenu de la morale dans sa forme parfaite.

On peut, en un premier temps, constater que le domaine éthique, celui dans lequel nous introduit la «morale par provision», comporte des illusions et des erreurs, ${ }^{15}$ et un disciple americain de Husserl ${ }^{16}$ a voulu étendre, à cau-

13 (4 août 1645 AT 3, 267) voir aussi lettre du 15 septembre 1645.

14 "Beatitas e scripturis : un exégète calviniste au 17ème siècle: Jacobus Kimedontius », La vie, la mort, la foi, le temps. Mélanges offerts à Pierre Chaunu, Paris, 1993, p. 397-401.

15 Max Scheller, "Sittlichkeit und ethische Werterkenntnis", Jahrbuch für Philosophische und Phänomenologische Forschung, V, 1922, p. 463 (rappelant les arguments de Dietrich von Hildebrand).

16 Herbert Spiegelberg, "Indubitables in Ethics : A Cartesian Meditation », Ethics (Chicago), LVIII, 194748 , p. $35-40$. 
se de cela, le doute à la morale, pour dégager de cette « mise entre parenthèses » les éléments indubitables qui permettent de la fonder. On peut objecter à cette expérience mentale deux arguments: d'abord, Descartes ne prétend pas dans le Discours que l'expérience morale échappe à l'erreur, c'est même pour cela qu'il ne propose qu'une morale « par provision », en en donnant les motifs. Ensuite, l'extension de l'épochè au domaine moral aboutit moins à fonder la morale qu'à dégager des éléments sociologiques, provenant d'un constat de valeurs. Il peut être intéressant d'envisager ce passage à l'état zéro de la décision morale, que Descartes écarte expressément. Les raisons que Descartes donne restent néanmoins valides: comment, dans ces conditions, peut-on même juger légitime de passer à l'examen attentif des fondements ? N'est-il pas nécessaire de conserver une rémanence («par provision ») qui permette d'initier l'agir de la réflexion ?

D'une toute autre portée me semble être la considération que le doute soit susceptible d'appartenir à la morale parfaite. Il convient ici de souligner qu'une telle hypothèse reflète la distinction, avancée par Descartes lui-même dans les Secondes réponses entre l'usage de la vie et la contemplation de la vérité:

Caeterum uelim ut hic memineritis me, circa ea quae licet amplecti uoluntati, accuratissime distinxisse inter usum uitae et contemplationem ueritatis. Nam, quod ad usum uitae attinet, tantum abest ut putem nullis nisi clare perspectis esse assentiendum, quin e contra nequidem uerisimilia puto esse semper expectanda, sed interdum e multis plane ignotis unum eligendum, nec minus firmiter tenendum, postquam electum est, quandiu nullae rationes in contrarium haberi possunt, quam si ob rationes ualde perspicuas fuisset electum ut in Dissertatione de Methodo pag. 26 explicui (IIae Resp., AT 7, 149). ${ }^{17}$

En commentant ce texte, Etienne Gilson a souligné qu'il existe un pouvoir de détermination intérieur à la volonté, et même, dans certains cas, un devoir de détermination, indépendamment de la connaissance intellectuelle. ${ }^{18}$ A partir de la réponse donnée par Descartes à Buitendijk en 1643 (sur la question de savoir s'il est possible de mettre en doute intellectuellement

17 Traduction de Ferd. Alquié : « je vous prie ici de vous souvenir que, touchant les choses que la volonté peut embrasser, j'ai toujours mis une très grande distinction entre l'usage de la vie et la contemplation de la vérité. Car, pour ce qui regarde l'usage de la vie, tant s'en faut que je pense qu'il ne faille suivre que les choses que nous connaissons très clairement, qu'au contraire je tiens qu'il ne faut pas même toujours attendre les plus vraisemblables, mais qu'il faut quelquefois, entre plusieurs choses tout à fait inconnues et incertaines, en choisir une et s'y déterminer, et après cela ne la pas croire moins fermement (tant que nous voyons point de raisons au contraire) que si nous l'avions choisie pour des raisons certaines et très évidentes, ainsi que je l'ai expliqué dans le Discours de la méthode page 26 [de l'éd. 1637, le texte de la «morale par provision»]» (Oeuvres, Éd. Alquié, t. 2, p. 574-575).

18 Commentaire du Discours de la méthode, p. 232. 
l'existence de Dieu), ${ }^{19}$ Gilson développe cette distinction, concluant à la stricte nécessité de la morale provisoire, qui doit apparaître non comme un expédiant, ni même comme une précaution facultative. Il poursuit:

«son fondement métaphysique premier est la distinction entre l'ordre du vrai et l'ordre du bien » (p. 233).

Nous touchons sans doute ici, par cette distinction, à la plus originale contribution de Descartes à la modernité. En distinguant ces deux ordres, Descartes ouvrait à la conscience morale les chemins et les traverses de l'aventure moderne. Quel type de certitude peut en effet qualifier une morale de perfection dans l'ordre du bien? Le bien le plus probable doit être choisi, pour éviter d'errer et de connaître le malheur. Nous ne considérons pas ici les conséquences techniques d'un tel point de vue dans les querelles morales du temps. ${ }^{20}$ Notre approche se réduit à la validité philosophique de cette distinction dans l'établissement d'une morale définitive.

Pour le dire autrement, lorsqu'un auteur anonyme prétendit établir un Art de vivre heureux à partir de très belles maximes de M. Descartes ${ }^{21}$, il dut le former «sur les idées les plus claires de la raison et du bon sens », ce qui revient à proposer une chimère composée par la raison et le bon sens tout à la fois, un mixte de pratique et de spéculatif dont on peut légitimement se demander s'il possède encore quelque cohérence.

En effet, d'une part, la plus parfaite morale présuppose «une entière connaissance des autres sciences » (AT IXb, 14), tandis que, d'autre part, Descartes, sans attendre, propose avec son traité des Passions une véritable morale, où la physique sert à «établir des fondements certains » (à Chanut, 15 juin 1646, AT 4, 441-442).

Qu'est-ce qui a permis à Descartes d'anticiper de la sorte sur son propre projet? Pour l'essentiel, me semble-t-il, sa conviction d'une « sagesse » qui correspond à l'idéal ultime de la vie morale.

« Les exigences de l'action,» écrit Geneviève Rodis-Lewis, « maintiennent une série de degrés de probabilité entre la totale incertitude et l'entière connaissance de ce qu'il faut faire: la plus parfaite morale, en tant qu'elle présupposerait ce savoir achevé, ne peut être qu'un idéal ».22

19 AT 4, 62.

20 Nous nous permettons de renvoyer à J.J.I. von Döllinger et F.H. Reusch Geschichte der Moralstreitigkeiten ...., (2ème éd., Nördlingen, 1889, reprint 1984 Scientia Vlg, Aalan) et nos articles sur le probabilisme de Caramuel (Revue de l'Histoire des Religions 3, 1983 p. 465-466 et dans les actes du colloque de Vigevano, Le meraviglie del probabile, Vigevano, 1990, p. 35-40).

21 L'art de vivre heureux, formé sur les idées les plus claires de la raison et du bon sens, et sur de très belles maximes de M. Descartes, 1ère éd; Paris, 1667 (souvent attribué à l'Oratorien Ameline, affirmation contestée par Henri Gouhier, La vocation de Malebranche, p. 71-72, n.).

22 La morale de Descartes, p. 118-119. 
Plus encore, dans cet idéale même, il reste une part d'indécision, voire de doute. Ce n'est plus le doute méthodique du Discours, mais une forme de doute permanent, rémanent, garantie de la résolution. C'est ainsi que je comprends le glissement, souvent analysé par les commentateurs, entre les trois règles de «morale par provision» émises dans le Discours de la méthode en 1637 et celles qu'il énonce à Elisabeth dans sa lettre du 4 août 1645 (AT IV, 265-266):

un chacun se peut rendre content de soi-même et sans rien attendre d'ailleurs, pourvu seulement qu'il observe trois choses, auxquelles se rapportent les trois règles de morale, que j'ai mises dans le Discours de la Méthode.

\section{Discours de la méthode}

La première était d'obéir aux lois et aux coutumes de mon pays, retenant constamment la religion en laquelle Dieu m'a fait la grâce d'être instruit dès mon enfance...

Ma seconde maxime était d'être le plus ferme et le plus résolu en mes actions que je pourrais, et de ne suivre pas moins constamment les opinions les plus douteuses, lorsque je m'y serais une fois déterminé, que si elles eussent été très assurées....

\section{Lettre de 1645}

[1] qu'il tache toujours de se servir, le mieux qu'il lui est possible, de son esprit, pour connaître ce qu'il doit faire ou ne pas faire en toutes les occurrences de la vie.

[2] qu'il ait une ferme et constant résolution d'exécuter tout ce que la raison lui conseillera, sans que ses passions ou ses appétits l'en détournent; et c'est la fermeté de cette résolution, que je crois devoir être prise pour la vertu, bien que je ne sache point que personne l'ait jamais ainsi expliquée; mais on l'a divisée en plusieurs espèces, auxquelles on a donné divers noms, à cause des divers objets auxquels elle s'étend.

Ma troisième maxime était de tâcher toujours plutôt à me vaincre que la fortune, et à changer mes désirs que l'ordre du monde; et généralement, de m'accoutumer à
[3] qu'il considère que, pendant qu'il se conduit ainsi, autant qu'il peut, selon la raison, tous les biens qu'il ne possède point sont aussi entièrement hors de son pou- 
croire qu'il n'y a rien qui soit entièrement en notre pouvoir, que nos pensées, en sorte qu'après que nous avons fait notre mieux, touchant les choses qui nous sont extérieures, tout ce qui manque de nous réussir est, au regard de nous, absolument impossible. ...» voir les uns que les autres, et que, par ce moyen, il s'accoutume à ne les point désirer; (...) si nous faisons toujours tout ce que nous dicte notre raison, nous n'aurons jamais aucun sujet de nous repentir, encore que les évènements nous fissent voir, par après, que nous nous sommes trompés, parce que ce n'est point par notre faute».

On perçoir le changement, d'une morale encore inspirée du stoïcisme à une sagesse humaine. C'est qu'entre temps, la constitution d'une métaphysique avait rendu permanente l'entreprise morale. Par provision, on peut bien se contenter de garder les lois de son pays et de se décider à rester résolu dans ses actions. Mais «la découverte métaphysique de l'homme» (F. Alquié) entraîne l'affirmation d'une morale définitive et imparfaite tout à la fois: Dieu seul peut totalement juger, dans l'adéquation du bien et du vrai, tandis que l'homme doit, pour suivre « la raison », « faire tout le mieux que notre entendement a pu connaître » (Passions de l'âme a. 146). Dieu seul peut connaître ce bonheur sans bornes qu'est l'adéquation du vrai et du bien; mais l'homme n'y reste pas totalement étranger pour autant.

L'expérience de la volonté me porte à me connaître comme image et ressemblance de Dieu :

sola est voluntas, sive arbitrii libertas, quam tantam in me experior, ut nullius majoris ideam apprehendam; adeo ut illa praecipue sit, ratione cujus imaginem quandam et similitudinem Dei me referre intelligo. (Meditatio IVa, AT 7, 57).

C'est cette image et ressemblance de Dieu, dont la volonté infinie que je porte en moi est le vestige, qui permet à l'homme d'avoir accès, de participer, à cette béatitude que Dieu seul peut connaître par l'adéquation du vrai et du bien. La preuve a contrario de la démarche se trouve dans la tentation de l'eritis sicut dii:

«nous pouvons venir à l'extravagance de souhaiter d'être dieux, et ainsi, par une très grande erreur, aimer seulement la divinité au lieu d'aimer Dieu» (AT 4, 608).

A contrario, c'est bien parce que nous avons en nous l'image et ressemblance de Dieu que nous pouvons commettre l'erreur de souhaiter d'être des dieux et de substituer à l'amour de Dieu l'amour de la divinité. Mais, par 
ailleurs, c'est parce que nous avons cette image et ressemblance que nous pouvons participer de cette béatitude à laquelle nous sommes appelés: «si la totale dépendance ontologique de l'homme à Dieu fait de chaque instant un choix de l'acte créateur dont l'éternité nous dépasse infiniment, c'est à notre niveau d'êtres temporels en situation, à l'occasion de tel ou tel évènement, que se manifeste notre choix » (Gen. Rodis-Lewis La morale de Descartes p.35). Le doute reste donc constitutif, de manière permanente, fondatrice, du projet moral; celui-ci peut devenir définitif, il ne cesse pas d'être prégnant de doute.

Il convient ici de rapprocher Descartes de Montaigne, chez qui la question «que sais-je?» est un moteur permanent de curiosité, c'est-à-dire l'essentiel de la démarche philosophique: la sagesse comme découverte, comme exercice et comme ascèse.

« Il n'est désir plus naturel que le désir de connaissance. Nous essayons tous les moyens qui nous y peuvent mener. Quand la raison nous faut, nous y employons l'expérience (...) qui est un moyen plus faible et plus vil, mais la vérité est chose si grande que nous ne devons dédaigner aucune entremise qui nous y conduise. La raison a tant de formes que nous ne savons à laquelle nous prendre; l'expérience n'en a pas moins » (Montaigne, Essais 3, 13 De L'expérience).

Le doute rémanent, à l'intérieur même d'une morale parfaite, signifie que la sagesse dans sa jouissance n'est pas limitée, ne connaître pas de rivage, et que le questionnement qui permet à l'homo viator l'enrichissement de bonheur que confèrent l'acquisition de la connaissance, son accroissement, est porté par cette rémanence du doute dans l'écartèlement paradoxal qui situe, de façon personnelle, mais aussi de façon historique et dans sa postérité, le questionnement de Descartes.

«L'un des points de ma morale est d'aimer la vie sans craindre la mort»: dans une lettre à Mersenne (9 janvier 1639, AT 2, 481), Descartes donne la clef intime de sa morale. Frappée comme une maxime, cette phrase permet, dans son analyse, de retrouver l'écartèlement des termes dans l'opposition entre «aimer» et «craindre», dans l'opposition entre «la vie» et «la mort». Aimer la vie sans craindre la mort, voilà qui, pour Descartes, constitue le cœur d'une sagesse quotidienne, qui n'est plus une morale «par provision», mais qui constitue le cœur définitif du projet moral. Et au cœur de ce projet un doute constitutif persiste. Ce n'est plus le doute méthodique, ce n'est pas le doute sceptique, mais c'est un doute épistémologique, à savoir la curiosité, source suprême de la sagesse. 


\section{BIBLIOGRAFIA}

[anônimo]. L'art de vivre heureux, formé sur les idées les plus claires de la raison et du bon sens, et sur de très belles maximes de M. Descartes. 1ère Èd; Paris, 1667.

Armogathe, Jean-Robert. «Beatitas e scripturis : un exégète calviniste au XVIIè siècle: Jacobus Kimedontius », La vie, la mort, la foi, le temps. Mélanges offerts à Pierre Сhаипи, Paris, 1993, p. 397-401.

Alquié, Ferdinand. La Découvert métaphysique de l'homme chez Descartes (Paris: Presses Universitaires de France, 1950).

Armogathe, Jean-Robert. «Probabilisme et casuistique : l'exemple de Caramuel (1606-1682) », Revue de l'Histoire des Religions 3, 1983, p. 465-466.

Descartes, René. Oeuvres de Descartes par Charles Adam et Paul Tannery, révisée et complétée par J. Beaude, P. Costabel et R. Rochot, 11 vols. Paris : J. Vrin, 1996.

Descartes, René. CEuvres Philosophiques, ed. F. Alquié, 3 vols., Paris : Bordas, 19631973.

Descartes, René. L'Entretien avec Burman, ed. J-M. Beyssade. Paris: PUF, 1981.

Gilson, Etienne. Commentaire du Discours de la Méthode. Paris : J. Vrin, 1925.

Gouhier, Henri. La vocation de Malebranche. Paris : J. Vrin, 1926.

Monet, Philibert. Inventaire des deux langues françoise et latine, assorti des plus utiles curiositez de l'un et de l'autre idiomes, Lyon, 1635, réimpr. de l'Èd. 1636 chez Slatkine, Genève (1973).

Rodis-Lewis, Geneviève. L'œuvre de Descartes. 2 vols., Paris, J. Vrin, 1971.

Rodis-Lewis, Geneviève. La morale de Descartes. Paris : PUF, 1957.

Scheller, Max. "Sittlichkeit und ethische Werterkenntnis", Jahrbuch für Philosophische und Phänomenologische Forschung, V, 1922.

Spiegelberg, Herbert. «Indubitables in Ethics : A Cartesian Meditation », Ethics (Chicago), LVIII, 1947-48.

Von Döllinger, J.J.I.et F.H. Reusch, Geschichte der Moralstreitigkeiten ...., (2è édition, Nördlingen, 1889, reprint 1984 Scientia Vlg, Aalan). 